\title{
ÉTUDE COMPARATIVE DE LA DIGESTION ANAÉROBIE ENTRE PULPES DE LA POMME DE CAJOU, BOUSE DE VACHE ET LEUR CODIGESTION
}

\author{
Faye Omar Kata ${ }^{1}$, Ndiaye Lat Grand ${ }^{1}$, Sarr Bassirou ${ }^{1,2}$. \\ ${ }^{1}$ Laboratoire de Chimie et de Physique des Matériaux, Université Assane Seck de Ziguinchor, Sénégal \\ ${ }^{2}$ Programme National de Biogaz du Sénégal, Ministère des Mines et de l'Industrie, 120, Cité ASECNA Liberté 6 Extension en face \\ Immeuble Ferdinand COLY, Dakar, Sénégal \\ *Corresponding author.E-mail: katafaye86@gmail.com
}

\author{
INFOS SUR L'A R T I C L E \\ Historique de l'article: \\ Reçu le : 26 novembre 2020 \\ Réçu en format revisé le : 20 février 2021 \\ Accepté le : 21 février 2021
}

Mots-Clés: Méthanisation, Codigestion,

Pulpes Pomme de Cajou, Bouse de Vache.

Keywords: Methanization, Codigestion, Cashew Apple Pulps, Cow dung.

\begin{abstract}
RE S U M E
La digestion anaérobie est un processus naturel biologique de dégradation de la matière organique en absence d'oxygène, pour la production du biogaz. L'étude réalisée porte sur la valorisation des déchets organiques dans la région de la Casamance. Elle consiste à évaluer la production de biogaz par les pulpes de la pomme de cajou (PPC), comparée à celle de la production par la bouse de vache (BV) et celle de la codigestion de ces deux substrats. Trois dispositifs expérimentaux ont été conçus d'une part, pour la détermination de la composition du biogaz et d'autre part, neuf dispositifs expérimentaux basés sur la méthode du déplacement du liquide ont été mis en œuvre pour la détermination du volume de biogaz. Les essais ont été triplet et les résultats basés sur la moyenne des mesures effectuées. Les expériences ont duré 40 jours dans un bain marie à $38^{\circ} \mathrm{C}$.

Les résultats montrent un volume cumulé de $5100 \mathrm{ml}$ de biogaz avec un pourcentage en méthane de $62,95 \%$ pour les $(\mathrm{PPC})$; la $(\mathrm{BV})$ présente un volume cumulé de biogaz de $3256 \mathrm{ml}$, composé de 58,52\% de méthane et enfin la codigestion des substrats mélangés ( $50 \%$ de pulpes de la pomme de cajou et $50 \%$ de bouse de vache), donne une production de $6982 \mathrm{ml}$ en volume cumulé contenant un pourcentage en méthane de $61,30 \%$. Ces résultats montrent l'importance de la codigestion sur l'amélioration des rendements de productions des substrats lors de la digestion anaérobie.
\end{abstract}

\section{INTRODUCTION}

La digestion anaérobie est une technologie qui a existé depuis plus d'un siècle, utilisé pour le traitement des eaux usées des usines, la stabilisation des boues organiques en excès pour la production de biogaz. Cette technologie est utilisée aussi pour le traitement des matières organiques solides (par ex. les déchets organiques et les cultures énergétiques) (Awe et $a l$., 2018 ; Esposito et al., 2012). De nos jours, de nombreux problèmes environnementaux, tels que la détérioration de la qualité de l'eau, les problèmes d'odeurs et d'eutrophisation se produisent en raison de l'utilisation irrationnelle des déchets (Nasir et al., 2012). Il s'avère donc nécessaire d'utiliser convenablement les déchets agricoles, ce qui peut réduire la pollution et atténuer la pénurie mondiale des ressources (Ning et al., 2020). Les pollutions environnementales et l'insécurité énergétique font partie des plus grands défis auxquels les êtres humains sont confrontés au $21^{\mathrm{e}}$ siècle (Raj et al., 2012). L'atténuation, des émissions de $\mathrm{CO}_{2}$ associé au réchauffement climatique, exige l'exploration des énergies alternatives afin de réduire la dépendance aux combustibles fossiles. En effet la production d'une énergie renouvelable rentable contribuerait à l'amélioration des niveaux de vie des pays en voie de développement (Garnier, 2014 ; Wu et al., 2009). À l'heure actuelle, les ressources en combustibles fossiles ne sont pas considérées comme durables en raison de la détérioration de l'environnement et de la consommation massive d'énergie (Chandra et al., 2012). Environ $90 \%$ de la consommation d'énergie provient des combustibles fossiles (Cheng et al., 2014); par exemple la combustion de combustibles fossiles pour produire de l'énergie est étroitement liée à l'émission de gaz à effet de serre dans l'atmosphère. Certaines recherches ont révélé que l'atmosphère terrestre reçoit plus de 15 millions de tonnes de dioxyde de carbone $\left(\mathrm{CO}_{2}\right)$ par an (Cheng et al., 2014). Tout ceci pouvant augmenter la concentration de ce gaz dans l'atmosphère ce qui peut augmenter la tendance du réchauffement climatique (Wilkie, 2005). Il est révélé que l'impact comparatif du $\mathrm{CH}_{4}$ sur le changement climatique est plus de 20 fois supérieure à celui du $\mathrm{CO}_{2}$ sur une période de cent ans (Stocker et al., 2014). Il est montré que le méthane peut piéger plus de rayonnement que le $\mathrm{CO}_{2}$ (Solomon, 2007 ; Scott et al., 2012). 
Pour répondre aux besoins en énergie afin de réduire sensiblement la pollution locale et l'effet de serre, et préserver l'environnement, la prospection et le développement de nouvelles sources d'énergie ont été entrepris notamment l'énergie issue de la biomasse (Inès, 2012). La digestion anaérobie est un processus naturel qui convertit la biomasse en énergie et les résidus de cette transformation constituent un engrais organique (Burke, 2001). Le biogaz produit par la digestion anaérobie se compose généralement de méthane (50 à $75 \%$ ), de dioxyde de carbone (45 à $20 \%$ ), de sulfure d'hydrogène et d'oligo-éléments. Pendant le processus de digestion anaérobie, les micro-organismes convertissent la matière organique complexe en composants chimiques plus simples. Environ $90 \%$ des composés organiques biodégradables peuvent être convertis en biogaz par le biais du processus de digestion anaérobie (Chandra et $a l$., 2012). La codigestion anaérobie est la digestion anaérobie d'au moins un mélange de deux substrats ayant des caractéristiques complémentaires, afin d'améliorer la production de biogaz (Mata-Alvarez et al., 2011). Dans un processus de codigestion, les déchets riches en protéines peuvent fournir la capacité tampon et une large gamme de nutriments, tandis que les déchets à haute teneur en carbone peuvent équilibrer le rapport $\mathrm{C} / \mathrm{N}$ pour tous les substrats caractérisés par un faible rapport $\mathrm{C} / \mathrm{N}$, diminuant le risque d'inhibition d'ammoniac (Hashimoto, 2086; Hills et al., 2081). Par conséquent, le principal problème $\mathrm{du}$ processus de codigestion réside sur l'équilibrage du rapport $\mathrm{C} / \mathrm{N}$, mais la bonne combinaison de plusieurs autres paramètres dans le mélange de cosubstrat, tels que les macros et micronutriments, le $\mathrm{pH} /$ alcalinité, les inhibiteurs/composés toxiques, les matières organiques biodégradables et les matières sèches sont également pertinents (Mata-Alvarez et al., 2011). Le fumier co-digéré avec d'autres substrats, y compris les cultures énergétiques, les déchets forestiers, les déchets botaniques ou agricoles, les déchets alimentaires et les déchets industriels, peut améliorer considérablement la production de biogaz (Crolla et al., 2011). Par ailleurs, le Sénégal est le $15^{\mathrm{eme}}$ exportateur mondial de noix de cajou avec une production de plus de 18000 tonnes par an selon l'étude du PADEC (Programme d'appui pour le développement de la Casamance) en 2018. Quatre régions principalement s'y investissent; Kolda, Ziguinchor, Sédhiou et Fatick. Alors que dans la région naturelle de la Casamance (Kolda, Ziguinchor, Sédhiou), chaque année après la campagne des noix de cajou, plus 342000 tonnes de pommes de cajou pressées ou non sont rejetées sans aucune valorisation, se dégradant ainsi dans la nature. Le but de cette étude est d'étudier et de comparer le potentiel de production de biogaz de deux substrats qui sont les pulpes de la pomme de cajou et la bouse de vache; ensuite les comparer avec leur codigestion en régime mésophile. La méthodologie adoptée réside sur l'évaluation de leurs cinétiques de productions et leurs compositions en biogaz tout en contrôlant l'évolution du $\mathrm{pH}$.

\section{MATERIEL ET METHODES}

\subsection{Préparation du substrat}

Dans cette étude, nous avons utilisé deux substrats, la bouse de vache collectée au niveau du lieu de stabulation des vaches, dans la région de Ziguinchor et les pulpes de la pomme de cajou provenant d'une unité de transformation des pommes en jus de fruit localisée à proximité de l'université Assane Seck de Ziguinchor (voir Photo 1).

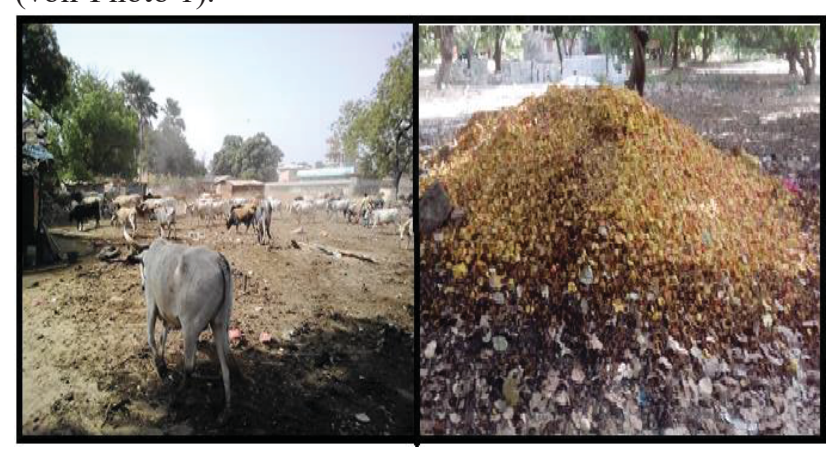

Photo.1. Lieux de collecte des substrats

La pomme de cajou, de caractéristique très acide avec un $\mathrm{pH}$ compris entre 4,2 à 5 , très fibreux, après collecte, a été prétraitée pour réduire les effets de ces deux caractéristiques qui sont des facteurs inhibiteurs lors de leur digestion anaérobie. Pour ce faire, elles ont été compostées à la température ambiante, sans retournement ni aération afin de provoquer une destruction des fibres par leur température interne et cela sur une durée allant d'une à deux semaines. Après cette étape, nous avons constaté que le $\mathrm{pH}$ des pommes s'est autorégulé jusqu'aux alentours de la neutralité, ce qui est important pour un substrat devant être méthanisé, car la plupart des méthanogènes ont un $\mathrm{pH}$ optimal compris entre 7 et 8 tandis que les bactéries formant l'acide ont souvent un optimum inférieur (Angelidaki et al., 2094). Si le pH des déchets à tester est en dehors de la plage optimale, et s'il n'y a pas suffisamment de capacité de mémoire tampon, le processus anaérobie peut être inhibé (Angelidaki et $a l$, 2014). La seconde étape de prétraitement consiste à broyer les pulpes de la pomme. Le broyage des pulpes a été effectué à l'aide d'un broyeur manuel, à une taille moyenne de particule inférieure à $2 \mathrm{~mm}$. Les différents substrats ont été échantillonnés comme suit :

$\rightarrow \quad 100 \%$ de la bouse de vache (100\% BV);

$\rightarrow 100 \%$ pulpes de la pomme de cajou (100\% PPC) ;

$\rightarrow 50 \%$ bouse de vache et $50 \%$ pulpe de la pomme de cajou $(50 \% \mathrm{BV}+50 \% \mathrm{PPC})$.

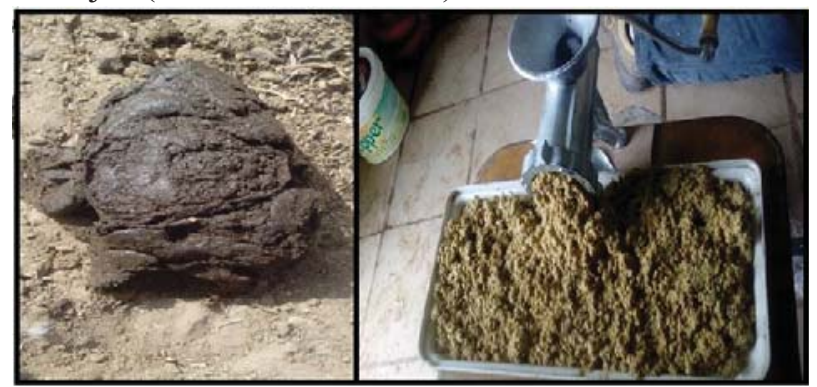

Photo.2. Les substrats utilisés 


\subsection{Procédure expérimentale}

Les substrats ainsi obtenus et l'inoculum ont été placés dans des bouteilles de capacité de 1,5 L pour la détermination de la cinétique de production des mélanges. Des réacteurs de $5 \mathrm{~L}$ ont servi à l'analyse de la composition du biogaz produit dans des conditions mésophiles $37{ }^{\circ} \mathrm{C}$ (Manyi-loh et al., 2013), suivant un rapport substrat/inoculum de 1/1. L'inoculum mésophile utilisé dans cette étude est prélevé dans notre réacteur de $10 \mathrm{~m}^{3}$ alimenté par la bouse de vache et installé sur la plateforme bioénergie de l'université Assane Seck de Ziguinchor. Les mélanges ont étés préparés de façon homogène. Au cours de la production du biométhane, il n'y a eu aucun ajout d'éléments nutritifs, y compris d'enzymes et de produits chimiques ; ceci afin d'évaluer la quantité et la qualité du biogaz produit par les substrats ainsi utilisés. Pour ce faire, la méthode de déplacement du liquide figure. 1 et celle du stock du biogaz produit figure. 2 ont été mis à contribution. Des triplicatas ont été réalisées pour chaque essai, les valeurs obtenues sont la moyenne des triplets sur chaque digesteur et ont été exprimées comme étant le volume (en $\mathrm{mL}$ ) de méthane produit par $\mathrm{g}$ de matière organique (MO) ajouté $\left(\mathrm{mLCH}_{4} / \mathrm{g} \mathrm{MO}\right)$.

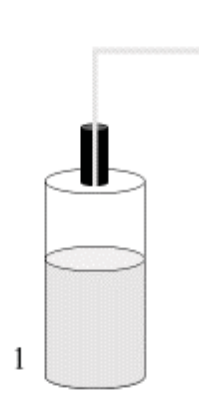

2 4

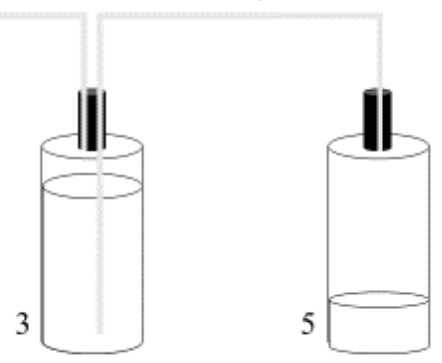

Fig.1. Méthode de déplacement du liquide

1 : Réacteur ;

2 : Tuyau de transport du biogaz;

3 : Bouteille remplie d'eau;

4 : Tuyau de transport de l'eau sortant de la bouteille ;

5 : Bouteille graduée pour la mesure de l'eau collectée. 4

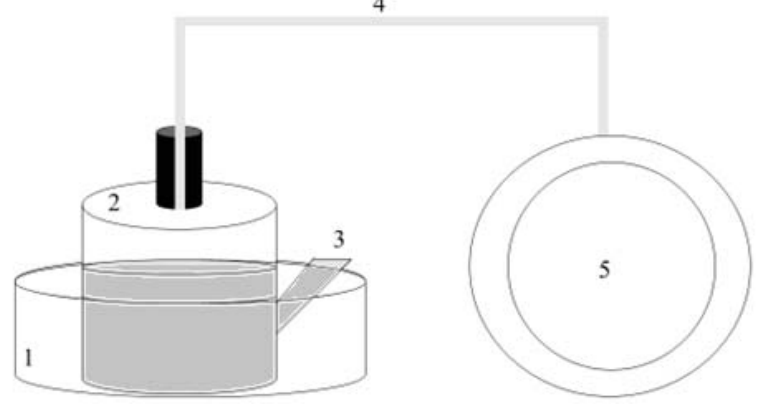

Fig.2. Dispositif expérimental de production du biogaz

1 : Bain marie à $37^{\circ} \mathrm{C}$;

2 : Réacteur de $5 \mathrm{~L}$;

3 : Buse de prélèvement ;

4 : Tuyau d'acheminement du biogaz vers la chambre à air ;

5 : Chambre à air pour la collecte du gaz produit.

\subsection{Méthodes analytiques}

L'expérimentation visant la détermination de la quantité de biogaz s'arrête lorsque la production de biogaz décroit de manière significative et ce à travers l'observation du dégonflement de la chambre à air.

Pour la détermination du pourcentage en matière sèche (\%MS) et de la teneur en humidité $(\% \mathrm{H})$; les substrats, ont été séchés dans une étuve ventilé à $105^{\circ} \mathrm{C}$ pendant 24 heures (Cheng et al., 2014; Park et al., 2014).

Pour la mesure de la teneur de la matière organique $(\% \mathrm{MO})$ et du pourcentage en matière minérale $(\% \mathrm{MM})$; les échantillons des différents substrats ont été séchés, puis broyés et enfin calcinés à $550{ }^{\circ} \mathrm{C}$ pendant 4 heures dans un four à moufle (Nikiema et al., 2015 ; Park et al., 2014).

Le pourcentage en carbone totale $(\% \mathrm{C})$ est déterminé par la méthode empirique (Afilal et al., 2014) en utilisant la formule de l'équation (1).

$$
\% \text { COT }=\frac{\% M O}{1,724}
$$

Le dosage de l'azote a été fait par la méthode de Kjedahl opérée suite à une minéralisation à l'acide sulfurique concentré et en présence d'un catalyseur de minéralisation $\left(\mathrm{K}_{2} \mathrm{SO}_{4}\right.$ et $\left.\mathrm{CuSO}_{4}\right)$; les composés azotés sont minéralisés en sulfate d'ammonium. L'ammoniac déplacé par la soude est entraîné par la vapeur de la solution et piégé dans une solution d'acide borique pour ensuite être dosé par une solution d'acide chlorhydrique (Danson et al., 2020; Nikiema et al., 2015). Cette méthode donne le pourcentage en azote $(\% \mathrm{~N})$ de l'échantillon par calcul à partir de l'équation (2).

$$
\% N=\frac{V \times T \times M_{N}}{m \times 1000} \times 100
$$

$\checkmark \quad \mathrm{V}$ : volume d'HCl

$\checkmark \quad \mathrm{T}$ : titrage de la solution $\mathrm{HCl}$

$\checkmark \quad \mathrm{M}_{\mathrm{N}}$ : masse molaire de l'azote

$\checkmark \mathrm{m}=$ masse de l'échantillon

La composition du biogaz produit sera aussi évaluée grâce à un analyseur de biogaz, modèle Optma7 Biogas.

\section{RESULTATS ET DISCUSSIONS}

\subsection{Caractérisation physico-chimique des substrats}

La présente étude vise à comparer la production du biogaz de nos trois échantillons (100\% pulpe de la pomme de cajou, $100 \%$ bouse de vache et leur codigestion constituée de $50 \%$ de pulpe de la pomme de cajou et 50 $\%$ de la bouse de vache). Le processus de la digestion anaérobie a été exécuté à l'état régulier où la température du processus s'effectue en milieu mésophilique (fixé à 37 ${ }^{\circ} \mathrm{C}$ par un bain marie) (Manyi-loh et al., 2013; Scully et al., 2005). Les caractéristiques physico-chimiques des substrats chargés sont mentionnées dans le tableau 1 cidessous. 
Tableau 1. Caractérisation physico-chimique des échantillons

\begin{tabular}{|c|c|c|c|}
\hline \multirow{2}{*}{ Paramètres } & \multicolumn{3}{|c|}{ Substrats } \\
\cline { 2 - 4 } & $\begin{array}{c}\text { Pomme de } \\
\text { cajou }\end{array}$ & $\begin{array}{c}\text { Bouse de } \\
\text { vache }\end{array}$ & $\begin{array}{c}50 \% \text { Bouse- } \\
50 \% \text { Pomme }\end{array}$ \\
\hline$\% \mathrm{MS}$ & 14,110 & 21,989 & 17,958 \\
\hline$\% \mathrm{H}$ & 85,890 & 78,011 & 82,042 \\
\hline$\% \mathrm{MO}$ & 98,586 & 80,412 & 88,949 \\
\hline$\% \mathrm{MM}$ & 1,414 & 20,588 & 11,051 \\
\hline$\% \mathrm{C}$ & 56,658 & 46,214 & 51,594 \\
\hline$\% \mathrm{~N}$ & 2,442 & 1,500 & 1,875 \\
\hline $\mathrm{C} / \mathrm{N}$ & 23,201 & 30,809 & 27,517 \\
\hline
\end{tabular}

Les substrats analysés présentent des teneurs en matières

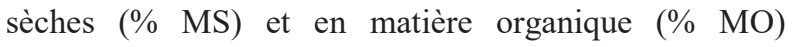
variables. Les caractéristiques des échantillons analysés, montrent une abondance de la matière organique dans les échantillons et cette dernière est considérée comme étant la partie du substrat qui est susceptible de se transformer en biogaz, y compris le méthane (Cheng et al., 2014). En outre, le rendement volumétrique de méthane peut être considérablement amélioré par une teneur élevée en matière organique des substrats chargés (Asam et al., 2011). Le pourcentage de la teneur en carbone de la pomme est assez élevé, ce qui indique que le substrat est favorable à la codigestion avec la bouse de vache qui a une teneur en carbone plus faible que celle de la pomme. Le rapport carbone/azote de la bouse de vache est de 30,809. Cependant, ce rapport $\mathrm{C} / \mathrm{N}$ n'est toujours pas approprié pour augmenter la production de méthane par digestion anaérobie car le rapport $\mathrm{C} / \mathrm{N}$ optimal pour la digestion anaérobie est dans l'intervalle 20 à 30 (Zhang et al., 2008). Ainsi, plus un substrat s'éloigne de cet intervalle, plus sa production en méthane diminue. Par conséquent, en co-digérant ce substrat avec les pulpes de la pomme qui ont un rapport $\mathrm{C} / \mathrm{N}$ égale à 23,201 , on peut améliorer les performances du processus de digestion anaérobie permettant de générer un rendement de production de méthane plus élevé. Par ailleurs, la codigestion des deux substrats donne un rapport carbone/azote de 27,517 qui se trouve dans la gamme donnée par la littérature. Par conséquent, le mélange entre $50 \%$ bouse de vache et $50 \%$ de pulpes de la pomme peut fournir des nutriments appropriés dans le cadre de la codigestion entre ces deux substrats.

\subsection{Variation du pH durant la digestion anaérobie des trois échantillons}

Le $\mathrm{pH}$ est le paramètre fondamental de la digestion anaérobie. Il est un indicateur très intéressant dans la stabilisation et le bon déroulement de la digestion anaérobie. Les procédés de digestion anaérobies sont fortement influencés par le $\mathrm{pH}$. La digestion anaérobie se déroule de façon optimale au voisinage de la neutralité, à $\mathrm{pH}$ égale à 7 avec une valeur optimale se trouvant dans l'intervalle de $\mathrm{pH}$ de 6,5 à 8,5 (Moletta, 2017), 7,0 à 7,5 (O'Flaherty et al., 2098).

En outre, la représentation de la variation de $\mathrm{pH}$ (figurig.3), nous permet d'observer les trois différentes étapes de la digestion anaérobie que sont (i) l'hydrolyse et l'acidogenése, (ii) l'acétogenèse et (iii) la méthanogenése.

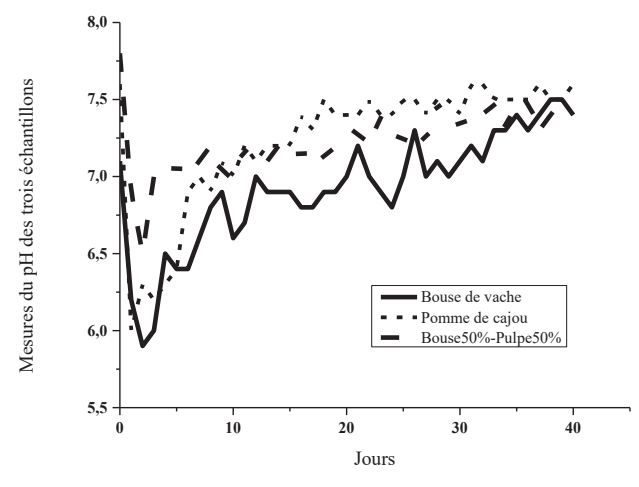

Fig. 3. Variation $d u p H$ des trois échantillons

L'allure générale des courbes commence par une diminution des valeurs du $\mathrm{pH}$ initial des trois échantillons passant de 7,8 à 5,9 durant les trois premiers jours. Cette diminution des valeurs du $\mathrm{pH}$ est due à la décomposition des polymères en monomères suivie par la fermentation de ces monomères en acides aminés; les sucres et les acides gras obtenus durant l'hydrolyse sont transformés en acides gras volatils (AGV) comme l'acétate, le butyrate, le propionate ou le lactate, en acides organiques autres (lactate) et alcools à l'aide de microorganismes acidogènes (Amani et $a l .$, 2010). Cette étape représente la première étape qui est l'étape d'hydrolyse et acidogenése.

La seconde étape est l'étape acétogenèse, elle a démarré dès le quatrième jour et elle se matérialise par l'autoajustement du $\mathrm{pH}$ qui tend vers la neutralité. Cela est dû à la formation d'acétate depuis les produits d'hydrolyse et d'acidogenése. Cette conversion peut s'effectuer suivant deux voies métaboliques grâce à des bactéries consommant soient les $\mathrm{AGV}$ soient le $\mathrm{CO}_{2}$ et l'hydrogène (Batstone et al., 2002). L'une des voies est la seconde voie métabolique d'acétogenèse, appelée homoacétogènes, l'autre est la méthanogenèse hydrogénoclastique. Il y a donc une association obligatoire entre les espèces produisant l'hydrogène et celles qui le consomment; c'est ce qu'on appelle une relation syntrophique (Delgenes et al., 2003; Nie et al., 2008 ; Schink, 2097).

La méthanogenèse est la dernière étape du processus de digestion anaérobie, elle est observée entre le $9^{\text {éme }}$ et le

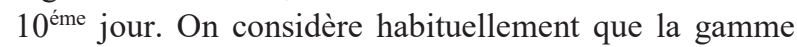
optimale de $\mathrm{pH}$ pour la digestion anaérobie se situe entre 6,7 et 7,3 (Braun, 2007 ; Stadtman et al., 2051). Lors de cette étape, l'acétate, l' $\mathrm{H}_{2}$ et le $\mathrm{CO}_{2}$ sont transformés en $\mathrm{CH}_{4}$ et en $\mathrm{CO}_{2}$. Cette réaction est réalisée par des archées de plusieurs types :

$\checkmark$ Les archées méthanogènes acétoclastes qui utilisent l'acétate comme substrat. Cette voie métabolique produit $70 \%$ du $\mathrm{CH}_{4}$ total dans la digestion anaérobie (Pavlostathis, 2091) et implique différents microorganismes comme Methanosaeta concilii ou Methanosarcina acetivorans (Amani et al., 2010).

$\checkmark$ Les archées méthanogènes hydrogénotrophes qui produisent du $\mathrm{CH}_{4}$ à partir du $\mathrm{CO}_{2}$ et de $1^{\prime} \mathrm{H}_{2}$. Cette voie correspond à $30 \%$ de la production $\mathrm{du}^{\mathrm{CH}_{4}}$ total 
dans la digestion anaérobie même si cette réaction est plus efficace énergiquement (Hattori et al., 2000) et peut être réalisée par Methanobacterium bryantii, Methanobrevibacter arboriphilus (Amani et al., 2010).

La figure 4 nous montre l'évolution journalière des trois échantillons étudiés au cours de la digestion anaérobie.

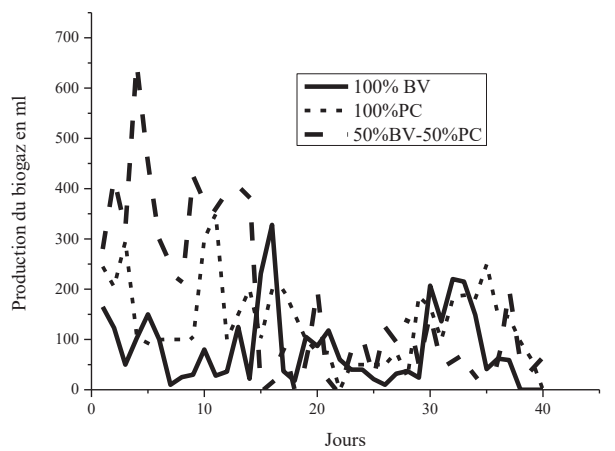

Fig. 4. Production journalière du biogaz des trois échantillons

La cinétique de production journalière de biogaz des trois échantillons a duré 40 jours jusqu'à ce que la production de biogaz ne soit plus observée. Pour tous les échantillons testés, la production de biogaz a démarré immédiatement dès le premier jour et les pics de production journalière de biogaz ont été observés après le premier jour sur l'ensemble des échantillons. Le taux de production de biogaz le plus élevé a été obtenu avec la codigestion entre $50 \%$ de bouse de vache et $50 \%$ de pulpes de pomme de cajou, avec un taux de production quotidien maximal de biogaz de $650 \mathrm{ml} / \mathrm{gMO} / \mathrm{j}$, le $4^{\text {éme }}$ jour. Après le cinquième jour, on a constaté une chute sur l'ensemble des productions des échantillons, coïncidant à la phase d'acétogenèse dont les vitesses réactionnelles sont généralement lentes et soumises à des problèmes d'inhibition liée à la présence de l'hydrogène qui modifie l'équilibre thermodynamique des réactions. Cependant, dès le neuvième jour on a constaté l'entrée dans la phase de méthanisation qui constitue l'étape de réduction finale du processus de méthanisation. Elle est considérée comme l'étape limitante dans le processus de dégradation des composés dissouts (Kaspar et al., 2078); durant cette phase, on a constaté de nouveau l'apparition de nouveaux pics de production sur l'ensemble des échantillons et le taux de production de biogaz le plus élevé est toujours obtenu par la codigestion $50 \%$ de bouse de vache avec $50 \%$ de pulpe de pomme de cajou, conduisant à une production journalière maximale de $425 \mathrm{ml} / \mathrm{gMO} / \mathrm{j}$ de biogaz. C'est après le vingtième jour, où on constate une production assez constante qui va jusqu'à la fin de l'expérimentation, au quarantième jour.

La cinétique de production cumulée des trois échantillons nous permette d'observer le classement quantitatif de la productivité (ou potentiel de production) des trois échantillons. La figure 5 ci-dessous donne une représentation du potentiel de production de biogaz de nos échantillons.

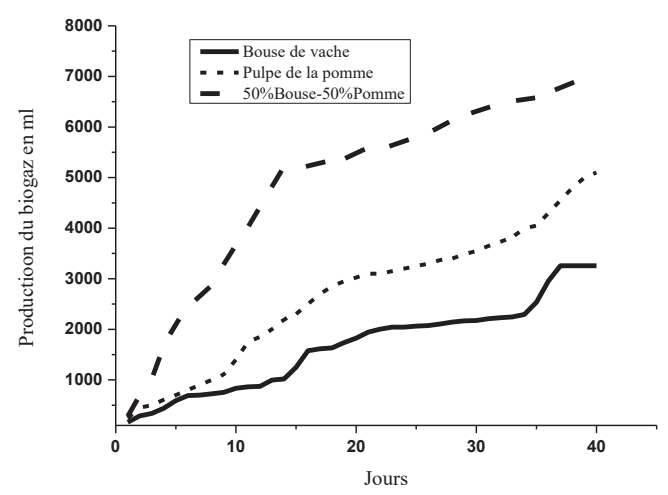

Fig. 5. Production cumulée du biogaz des trois échantillons

Après 40 jours de digestion anaérobie, la cinétique de production des trois échantillons montre que la codigestion de l'échantillon ( $50 \%$ bouse de vache et 50 $\%$ de pulpes de la pomme de cajou) produit la plus grande quantité de biogaz qui atteint un maximum de $6982 \mathrm{ml}$ de biogaz, vient en deuxième position l'échantillon composé de $100 \%$ pulpes de pomme de cajou avec une production de $5100 \mathrm{ml}$ de biogaz et enfin l'échantillon à $100 \%$ bouse de vache produisant $3256 \mathrm{ml}$ de biogaz. Quantitativement, la production de l'échantillon (50\% bouse de vache et $50 \%$ pulpes de la pomme de cajou) est meilleure. Par ailleurs, comparativement à la charge organique introduite dans les digesteurs qui est de l'ordre de $333 \mathrm{gMO} / \mathrm{L}$, il faut noter que les différents volumes produits demeurent intéressants. Les performances des digesteurs dépendent avant tout de la composition du substrat utilisé. L'analyse de la productivité qualitative du biogaz comprend à la fois une détermination de la composition du biogaz produit et de son pouvoir calorifique. Le tableau 2 ci-dessous nous donne la composition du biogaz et les pouvoirs calorifiques de nos trois échantillons.

Tableau 2. Composition du biogaz et pouvoirs calorifiques des échantillons testés

\begin{tabular}{|c|c|c|c|}
\hline & \multicolumn{3}{|c|}{ Substrats } \\
\cline { 2 - 4 } & $\begin{array}{c}\text { Bouse de } \\
\text { vache }\end{array}$ & $\begin{array}{c}\text { Pomme } \\
\text { de } \\
\text { cajou }\end{array}$ & $\begin{array}{c}50 \% \text { pulpe- } \\
50 \% \text { bouse }\end{array}$ \\
\hline $\mathrm{CH}_{4}(\%)$ & 58,52 & 62,95 & 61,30 \\
\hline $\mathrm{CO}_{2}(\%)$ & 39,24 & 32,72 & 34,75 \\
\hline $\mathrm{PCS}\left(\mathrm{kcal} / \mathrm{Nm}^{3}\right)$ & 5532 & 6568 & 6329 \\
\hline
\end{tabular}

La composition du biogaz produit par nos trois échantillons a été obtenue à l'aide d'un analyseur de biogaz (modèle OPTIMA BIOGAS7). Cet analyseur permet une détermination de la composition du biogaz produit et son pouvoir calorifique supérieur (PCS).

La qualité du biogaz produit est évaluée essentiellement par le pourcentage de méthane $\left(\mathrm{CH}_{4}\right)$ qu'il contient. Un biogaz est d'autant meilleur que son pourcentage en méthane est élevé (Sadak et al., 2011). D’après les résultats du tableau 2 , du point de vue qualitatif, les pulpes de la pomme de cajou représentent le meilleur substrat avec un pourcentage de méthane de $62,95 \%$, vient en deuxième position la codigestion de $50 \%$ de bouse de vache avec $50 \%$ de pulpe de pomme de cajou 
avec un pourcentage en méthane de $61,30 \%$ et enfin la bouse de vache avec un pourcentage de méthane de 58,52 $\%$. Le tableau 3 ci-dessous nous permet de faire une étude comparative globale entre nos trois échantillons.

Tableau 3. Étude comparative des trois échantillons

\begin{tabular}{|c|c|c|c|}
\hline \multirow{2}{*}{ Substrats } & \multicolumn{3}{|c|}{ Paramètres } \\
\cline { 2 - 4 } & $\mathrm{CH}_{4}(\%)$ & $\mathrm{C} / \mathrm{N}$ & $\begin{array}{c}\text { Volume de } \\
\text { biogaz (ml) }\end{array}$ \\
\hline $\begin{array}{c}\text { Bouse de } \\
\text { vache }\end{array}$ & 58,52 & 30,809 & 3256 \\
\hline $\begin{array}{c}50 \% \text { pulpe- } \\
50 \% \text { bouse }\end{array}$ & 61,30 & 27,517 & 6982 \\
\hline $\begin{array}{c}\text { Pulpe de la } \\
\text { pomme de } \\
\text { cajou }\end{array}$ & 62,95 & 23,201 & 5100 \\
\hline
\end{tabular}

L'intérêt énergétique du biogaz produit à partir des résidus de d'origine végétale et animale réside dans sa teneur plus ou moins importante en méthane qui lui confère un pouvoir énergétique certain. Rappelons que la proportion de méthane dans le biogaz se situe entre 55 et $75 \%$ (Igoni et al., 2008). En comparant nos différents échantillons, entre eux, les pulpes de la pomme de cajou représentent le meilleur substrat du fait de leur pourcentage en méthane plus élevé. Par ailleurs, le potentiel méthanogènes ou la production cumulée du biogaz produit lors de la codigestion $50 \%$ de bouse de vache et $50 \%$ de pulpe de pomme de cajou reste le plus élevé que celui obtenu avec les pulpes prises seules. Des observations similaires ont également été signalées par (Hills, 2079) pour le fumier de bovins laitiers, démontrant que la plus grande production de méthane a été réalisée lorsque le rapport $\mathrm{C} / \mathrm{N}$ était ajusté à 25 en utilisant du glucose (Hills, 2081). Dans cette étude, le rapport C/N produisant le meilleur rendement en méthane est de 23,201 proches de l'optimum qui est 25. L'optimisation du rapport $\mathrm{C} / \mathrm{N}$ des substrats par codigestion de différents déchets ayant des valeurs de $\mathrm{C} / \mathrm{N}$ très différentes, peut améliorer considérablement l'efficacité de la production de biogaz. Bien que de nombreuses études indiquent que les rapports $\mathrm{C} / \mathrm{N}$ optimaux à la digestion anaérobie sont de 25 à 30 (Marchaim et al., 2093; Yen et al., 2007). Dans notre cas, la codigestion de $50 \%$ de bouse de vache et 50 $\%$ de pulpes de pomme de cajou a amélioré le rapport $\mathrm{C} / \mathrm{N}$ de la bouse de vache qui était de 30,809 à 27,517 par augmentation du pourcentage de production de méthane de la bouse de vache qui passe de 58,52 à 61,30\% avec un rendement de production de biogaz deux fois plus grand que celle de la production de la bouse de vache prise seule.

\section{CONCLUSION}

Cette étude a démontré un effet interactif entre le rapport $\mathrm{C} / \mathrm{N}$, la production de biogaz et sa composition sur la performance de la codigestion anaérobie entre les pulpes de la pomme et la bouse de vache.

Les résultats de l'étude montrent que la pomme de cajou présente un rapport de $\mathrm{C} / \mathrm{N}$ égale à 23,201 , celle de la bouse de vache vient avec un rapport de 30,809 et enfin la codigestion de $50 \%$ pulpe-50\% bouse avec un rapport de 27,517. Nos résultats suggèrent que le rapport $\mathrm{C} / \mathrm{N}$ du substrat compris entre 20 et 25 (pulpe de la pomme de cajou) produit le meilleur rendement en méthane (62,95 $\%$ ). Cependant, la production cumulée du biogaz la plus importante est observée à un ratio $\mathrm{C} / \mathrm{N}$ se trouvant dans l'intervalle 25 à $30(50 \%$ pulpe de la pomme et $50 \%$ de bouse) soit $6982 \mathrm{~mL}$ de biogaz.

En sommes, nous pouvons dire que la codigestion des substrats a considérablement amélioré la production du biogaz en augmentant la composition en méthane de la bouse de vache et la production cumulée en biogaz des pulpes de la pomme de cajou.

\section{REMERCIEMENTS}

Les auteurs remercient le Programme National de Biogaz du Sénégal (PNB) et World Federation of Scientists (WFS), pour leurs supports.

\section{REFERENCES}

Afilal, M. E., Elasri, O., and Merzak. Z. 2014. "Caractérisations Des Déchets Organiques et Évaluation Du Potentiel Biogaz (Organic Waste Characterization and Evaluation of Its Potential Biogas)." J. Mater. Environ. Sci. 5(4): 1160-69.

Ahammad, S. Z.; Gomes, J.; Sreekrishnan, T. R. 2008. "Wastewater Treatment ForproductionofH2S-Free Biogas." Journal of Chemical Technology \& Biotechnology 83: 116369.

Amani, T., M. Nosrati, and T. R. Sreekrishnan. 2010. "Anaerobic Digestion from the Viewpoint of Microbiological, Chemical, and Operational Aspects - A Review." Environmental Reviews 18(1): 255-78.

Angelidaki, I., and B. K. Ahring. 2094. "Anaerobic Thermophilic Digestion of Manure at Different Ammonia Loads: Effect of Temperature." Water Research 28(3): 727 31.

Asam Zaki ul Zaman, Tjalfe Gorm Poulsen, Abdul-Sattar Nizami, Rashad Rafique, Ger Kiely, Jerry D. Murphyet. 2011. "How Can We Improve Biomethane Production per Unit of Feedstock in Biogas Plants?” Applied Energy 88(6): 2013-18. http://dx.doi.org/10.1016/j.apenergy.2010.12.036.

Awe Olumide Wesley, Yaqian Zhao, Ange Nzihou, Doan Pham Minh, Nathalie Lyczko. 2018. "Anaerobic Co-Digestion of Food Waste and FOG with Sewage Sludge-Realising Its Potential in Ireland." International Journal of Environmental Studies 75(3): 496-517.

Batstone, D. J., Keller, J., Angelidaki, I., Kalyuzhnyi, S.V., Pavlostathis, S.G., Rozzi, A., Sanders, W.T.M., Siegrist, H. and Vavilin, V.A. "The IWA Anaerobic Digestion Model No 1 (ADM1)." Water science and technology: a journal of the International Association on Water Pollution Research 45(10): 65-73.

Braun, Rudolf. 2007. “Anaerobic Digestion: A Multi-Faceted Process for Energy, Environmental Management and Rural Development." Improvement of Crop Plants for Industrial End Uses: 335-416.

Burke, Dennis A. 2001. "Dairy Waste Anaerobic Digestion Handbook: Options for Recovering Beneficial Products From Dairy Manure." Environmental Energy Company: 57.

Chandra, R., H. Takeuchi, and T. Hasegawa. 2012. "Methane Production from Lignocellulosic Agricultural Crop Wastes: A Review in Context to Second Generation of Biofuel 
Production." Renewable and Sustainable Energy Reviews 16(3): 1462-76. http://dx.doi.org/10.1016/j.rser.2011.11.035.

Cheng, Jay J, Zhimin Liu, Jorge Gontupil, and O-seob Kwon. 2014. "Anaerobic Co-Digestion of Rice Straw and Digested Swine Manure with Different Total Solid Concentration for Methane Production.” Int J Agric \& Biol Eng 7(6): 79-90.

Crolla, A., Kinsley, C., Sauvé, T., and Kennedy. K. 2011. "Anaerobic Digestion of Manure with Various CoSubstrates." Wastewaster Centre: 3-6.

Danson Donald, Obed Ehoneah, Isaac Ayensu, Abena Amponsaa Brobbey, Joseph Kwasi Adu, Samuel Oppong Bekoe. 2020. "Determination of Tryptophan Content in Hausa Koko ( Spicy Millet Porridge ) : A Ghanaian Beverage." International Journal of Phytopharmacy 9(4): 5287.

Delgenes, J. P., V. Penaud, and R. Moletta. 2003. "Pretreatments for the Enhancement of Anaerobic Digestion of Solid Wastes." ChemInform 34(13).

Esposito, G., Frunzo, L., Giordano, A., Liotta, F., Panico, A., Pirozziet F. 2012. "Anaerobic Co-Digestion of Organic Wastes." Reviews in Environmental Science and Biotechnology 11(4): 325-41.

Garnier, Gil. 2014. "Grand Challenges in Chemical Engineering." Frontiers in Chemistry 2(APR): 1-3. doi: 10.3389/fchem.2014.00017.

Hashimoto, Andrew G. 2086. "Ammonia Inhibition of Methanogenesis from Cattle Wastes." Agricultural Wastes 17(4): 241-61.

Hattori, Satoshi, Yoichi Kamagata, and Satoshi Hanada. 2000. "A Strictly Anaerobic, Thermophilic, Syntrophic AcetateOxidizing Bacterium.” International Journal of Systematic and Evolutionary Microbiology (50): 1601-9.

Hills, David J. 2079. "Effects of Carbon: Nitrogen Ratio on Anaerobic Digestion of Dairy Manure." Agricultural Wastes 1(4): 267-78.

Hills, David J., and David W. Roberts. 2081. "Anaerobic Digestion of Dairy Manure and Field Crop Residues." Agricultural Wastes 3(3): 179-89.

Igoni A. H., Ayotamuno M. J., Eze C. L., Ogaji S. O. T., Probert S. D., 2008. Designs of anaerobic digesters for producing biogas from municipal solid-waste. Applied Energy, vol. 85, pp. 430-438.

Inès, M'Sadak Youssef et Zoghlami Rahma. 2012. "Biométhanisation Industrielle Avicole En Tunisie SemiAride." Algerian journal of arid environment 2: 16-27.

Irini Angelidaki; Wendy Sanders. 2014. "Assessment of the Anaerobic Biodegradability of Macropollutants." Reviews in Environmental Science and Bio/Technology.

Kaspar, H. F., and K. Wuhrmann. 2078. "Kinetic Parameters and Relative Turnovers of Some Important Catabolic Reactions in Digesting Sludge." Applied and Environmental Microbiology 36(1): 1-7.

Manyi-loh, Christy E, Sampson N Mamphweli, Edson L Meyer, and Anthony I Okoh. 2013. "Microbial Anaerobic Digestion (Bio-Digesters) as an Approach to the Decontamination of Animal Wastes in Pollution Control and the Generation of Renewable Energy." Int. J. Environ. Res. Public Health: 4390-4417.

Marchaim, Uri, and Carsten Krause. 2093. "Propionic to Acetic Acid Ratios in Overloaded Anaerobic Digestion."
Bioresource Technology 43(3): 205-203.

Mata-Alvarez, Joan, Joan Dosta, Sandra Macé, and Sergi Astals. 2011. "Codigestion of Solid Wastes: A Review of Its Uses and Perspectives Including Modeling." Critical Reviews in Biotechnology 31(2): 99-111.

Moletta, René. 2017. "Méthanisation de La Biomasse." Techniques de l'Ingénieur 33(0): 1-21.

Nasir, Ismail M., Tinia I. Mohd Ghazi, and Rozita Omar. 2012. "Anaerobic Digestion Technology in Livestock Manure Treatment for Biogas Production: A Review." Engineering in Life Sciences 12(3): 258-69.

Nie, Yan Qiu, He Liu, Guo Cheng Du, and Jian Chen. 2008. "Acetate Yield Increased by Gas Circulation and Fed-Batch Fermentation in a Novel Syntrophic Acetogenesis and Homoacetogenesis Coupling System." Bioresource Technology 99(8): 2989-95.

Nikiema Mahamadi, Joseph B. Sawadogo, Marius K. Somda, Desiré Traore, Dayéri Dianou et Alfred S. Traoreet. 2015. "Optimisation de La Production de Biométhane à Partir Des Déchets Organiques Municipaux Optimization of Biomethane Production from Municipal Solid Organic Wastes." International Journal of Biological and Chemical Science 9: 2743-56.

Ning Jing, Mingdian Zhoua, Xiaofang Pana, Chunxing Lic, Nan Lva, Tao Wanga, Guanjing Caia, Ruming Wanga, Junjie Lia, Gefu Zhu. 2020. "Simultaneous Biogas and Biogas Slurry Production from Co-Digestion of Pig Manure and Corn Straw: Performance Optimization and Microbial Community Shift." Bioresource Technology 282(2016): 37-47. https://doi.org/10.1016/j.biortech.2020.02.122.

O'Flaherty, Vincent, Thérèse Mahony, Ronan O'Kennedy, and Emer Colleran. 2098. "Effect of PH on Growth Kinetics and Sulphide Toxicity Thresholds of a Range of Methanogenic, Syntrophic and Sulphate-Reducing Bacteria." Process Biochemistry 33(5): 555-69.

Park, Nathan D, Ronald W Thring, and Steve S Helle. 2014. "Comparison of Methane Production by Co-Digesting Fruit and Vegetable Waste with First Stage and Second Stage Anaerobic Digester Sludge from a Two Stage Digester." Water Science \& Technology.

Pavlostathis, S. G. E. Giraldo-Gomez. 2091. "Kinetics of Anaerobic Treatment: A Critical Review." Critical Reviews in Environmental Control 21(5-6): 411-90.

Raj, Bharat, and Onkar Singh. 2012. "Global Trends of Fossil Fuel Reserves and Climate Change in the 21st Century." Fossil Fuel and the Environment.

Sadak, Y M, A Ben M Barek, and R I Zoghlami S Baraket. 2011. "Caractérisation Des Co-Produits de La Biométhanisation Appliquée à La Biomasse Animale." Revue des Energies Renouvelables 14: 343-56.

Schink, B. 2097. "Energetics of Syntrophic Cooperation in Methanogenic Degradation." Microbiology and molecular biology reviews : MMBR 61(2): 262-80.

Scott, Anderson T., and Baylee L. Campbell. 2012. "Emissions of Methane and Nitrous Oxide from Natural Sources." Emissions of Methane and Nitrous Oxide from Natural Sources: 1-225.

Scully, C, G Collins, and V O Flaherty. 2005. "Assessment of Anaerobic Wastewater Treatment Failure Using Terminal Restriction Fragment Length Polymorphism Analysis." 
Journal of Applied Microbiology: 1463-71.

Solomon, Susan. 2007. "Climate Change 2007: The Physical Science Basis : Contribution of Working Group I to the Fourth Assessment Report of the Intergovernmental Panel on Climate Change." book Cambridge by Cambridge university press: 104. https://lib.ugent.be/catalog/rug01:001333500.

Stadtman, Thressa C., and H. A. Barker. 2051. "Studies on the Methane Fermentation X." Journal of Bacteriology 62(3): 269-80.

Stocker, T. F.; Qin, D.; Plattner, G.-K.; Tignor, M. M. B.; Allen, S. K.; Boschung, J.; Nauels, A.; Xia, Y.; Bex, V.; Midgley, P. M. (éds.) (2014). Climate Change 2013: The Physical Science Basis. Contribution of Working Group I to the Fifth Assessment Report of IPCC the Intergovernmental Panel on Climate Change. Cambridge: Cambridge University Press https://boris.unibe.ch/id/eprint/71452
Wilkie, A. C. 2005. “Anaerobic Digestion of Dairy Manure: Design and Process Considerations." Natural Resource, Agriculture, and Engineering Service,: 301-12.

Wu Changhua, Crescencia Maurer, Yi Wang, Shouzheng Xue, and Devra Lee Davis. 2009. "Water Pollution and Human Health in China Environmental." Environmental Health 107(4): 251-56.

Yen, Hong-wei, and David E Brune. 2007. "Anaerobic CoDigestion of Algal Sludge and Waste Paper to Produce Methane." Bio resource Technology 98: 130-34. doi:10.1016/j.biortech.2005.11.010

Zhang Panyue, Guangming Zeng, Guangming Zhang, Yin Li, Bibo Zhang, Maohong Fan. 2008. "Anaerobic Co-Digestion of Biosolids and Organic Fraction of Municipal Solid Waste by Sequencing Batch Process.” Fuel Processing Technology 89(4): 485-89. 


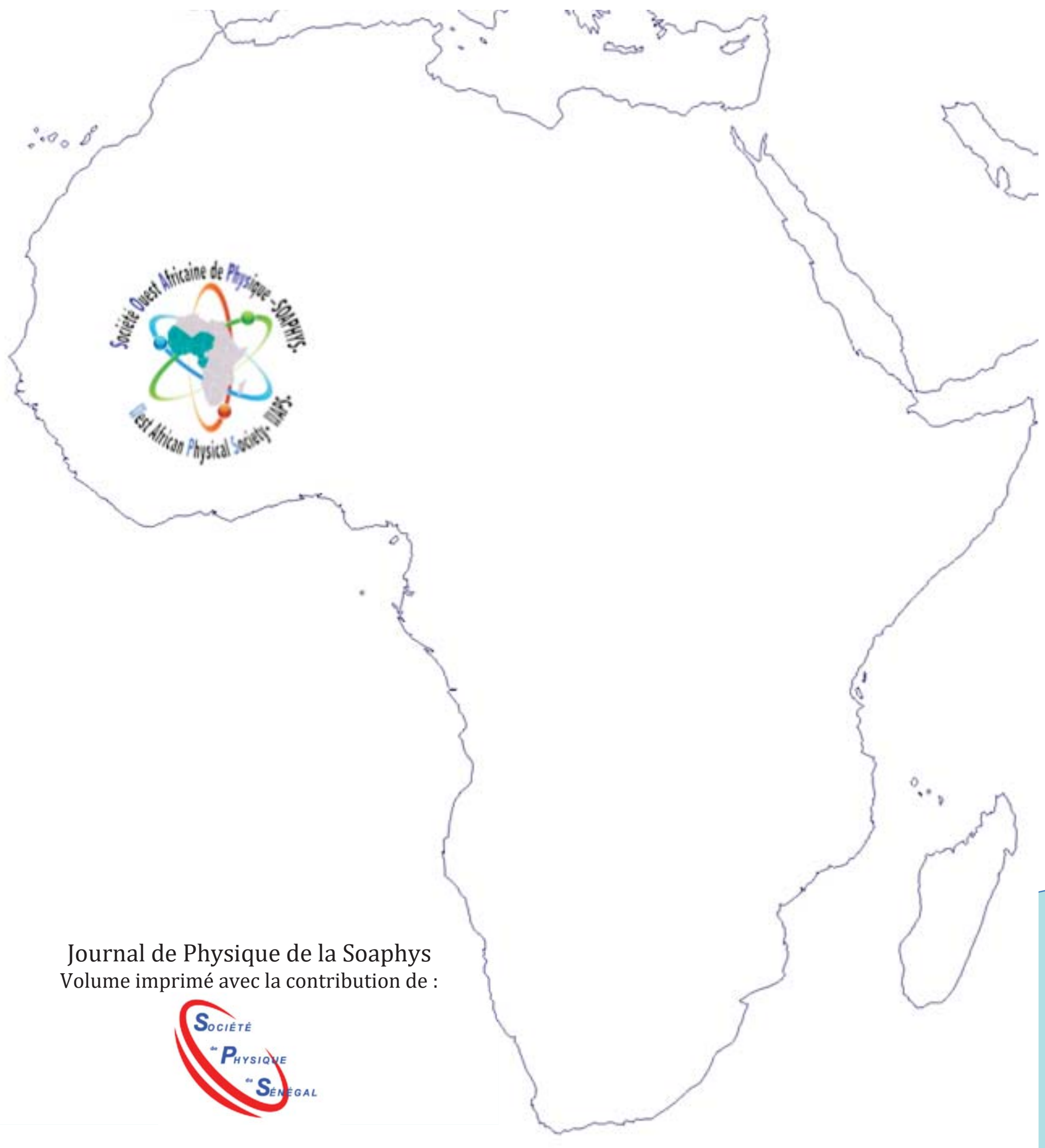

